\title{
CONTROL AND INITIAL OPERATION OF THE EERMILAB BO LOW B INSERTION
}

D. A. Finley, R. P. Johnson and F. Willeke

Fermilab[*], P.0.Box 500, Batavia, Illinois, 60510

\section{Abstract}

The operation of the Fermilab 80 low 3 insertion involves the coordinated control of the 4 strings of guadrupoles of the insertion itself along with several magnetic correction elements ( 20 dipolos for the cosed orbit, 2 quad circuits for betatron tunes, 1 skew quad circuit for coupling, and 2 sextupole circuits for chromaticity). When the beam is stored at high energy, these elements must correct the errors induced by the strong superconducting quadrupoles of the insertion as the opties are smoothly changed from the fixed target configuration to the low $B$ state. The techniques and control programs for these manipulations and initial tests using a single coasting beam are cescribed.

\section{Introduction}

The highest pogsible luminosity is needed to see rare processes. To increase the luminosity of an antiproton-proton collider using bunched beams, one can increase the beam currents and/or decrease the transverse beam size by either reduoing the emittance or $\beta$ value at the interaction point. Higher antiproton currents are difficult to achieve. Increased beam intersicy is undesireable in general because of possible collective instabilities and, specifically for a superconducting machine, because of increased risk of quenches due to beam loss. As well, the normalized emittances of the stored beams are properties of the injentor accelerators and are difficult to reduce. Most important in the consideration of which methods to increase the luminosity are are most effective is the beam-beam tune shift. Increased beam currents, lower emittance, and number of bunches each make the beam-beam tune shift worse. This tune shift, or tune spread, is not affected by a local change in the $B$ function.

Lowering the $B$ value at the interaction region, then, is the most effective way to increase the luminosity. This is true up to the point where the $B$ value is smaller than the longitudinal beam dimension or the large beam at a corresponding high $\beta$ region encounters regions of poorer field qualiby leading to a shorter beam lifetime.

The original studies of low $\beta$ insertions in the Tevatron and the particular design we have used are described in another paper at this conference. [1] Higher-current versions of the Tevatron quadrupoles determined the particular insertion used at the $B O$ straight section; this and the special features of the installation are described in another paper at this conference. [2]

\section{Insertion Description}

Figure 1 shows a schematic of the Bo straight section of the Tevatron. In the low $a$ configuration there are 4 new electrical circuits with quadrupoles similar to those of the regular cell but with a superconducting cable capable of higher maximum currents $(\epsilon$ $\mathrm{KA}$ vs $4.5 \mathrm{kA}$ ) and fields. The cable has $20 \mathrm{micron}$ diameter Niti filaments (compared to 8 microns) and $\mathrm{Cu} / \mathrm{NiTi}$ of 1.3 (vs. 1.8 ) by volume. The Q1 circuit has two $1.68 \mathrm{~m}$ long quadrupoles which replace the $0.81 \mathrm{~m}$ long quadrupoles in the regular Tevatron lattice. Thus, the Q1 circuit is the only one of the four which is on when the Tevatron is operating for fixed target physics. The Q1 circuit is also unusual in that its polarity changes at one point in the transition to the low $\beta$ configuration.

The Q2 circuit contains two $4.57 \mathrm{~m}$ long quadrupoles, the $Q 3$ circuit consists of four $3.66 \mathrm{~m}$ quadrupoles, and the $Q 4$ circuit uses two 3.56 m quadrupoles. The number of circuits is of particular importance for a super-conducting low $\beta$ insertion because each transition from the normal conductor to the cold region is an additional load on the refrigeration system. At the CFRN SPS where this is not an additional constraint, a similar low $\beta$ insertion requires 15 independently controlled supplies. [3]

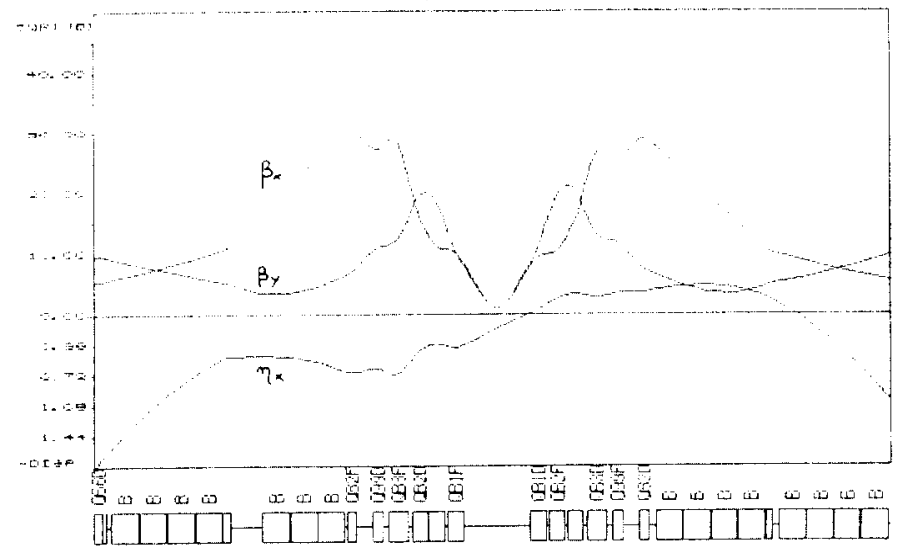

Figure 1 Schematic of the low $\beta$ insertion showing the lattice functions with all magnets energized. The $\beta$ values at the interaction point are approximately 0.8 $m$ in each plane. Maximum $\beta$ values in the insertion are close to $1000 \mathrm{~m}$. This large value prohibits injection in the low $B$ optics. Therefore an adiabatic change from the fixed target or injection lattice to the low $\beta$ configuration while the beam is stored at high energy is necessary.

\section{The Squeeze}

The transition from the extraction optios to the low $B$ configuration is called the squeeze. In order to maintain optimum performance of the Tevatron as a fixed target machine, an important design criterion for the insertion was to be able to start with with the Tevatron in its fixed target lattice configuration and to continuously vary the lattice until the low $\beta$ is reached. 'This requirement manifests itselt' in 28 intermediate optical configurations providing constant tunes and controlled amplitude functions during the transition to the low $B$ optics. The values for the quadrupole and correction element currents between these steps are interpolated linearly. Figure 2 shows the current programs for the four new circuits during the process.

Special dipole correction elements next to the low $\beta$ quadrupoles are needed in order to correct for misalignments of these elements as locally as possible to avoid magnified errors in the closed orbit around 
the ring. The extra focusing also causes changes in the betatron tunes which are compensated using the regular Tevacron correction quadrupoles. Including skew quadrupoles and chromaticity correcting sextupole circuits, some 29 function generators need to be changed together for each of the 28 steps between the iritial ard final configurations.

Ba QUADS for file 8 10/01/84:147

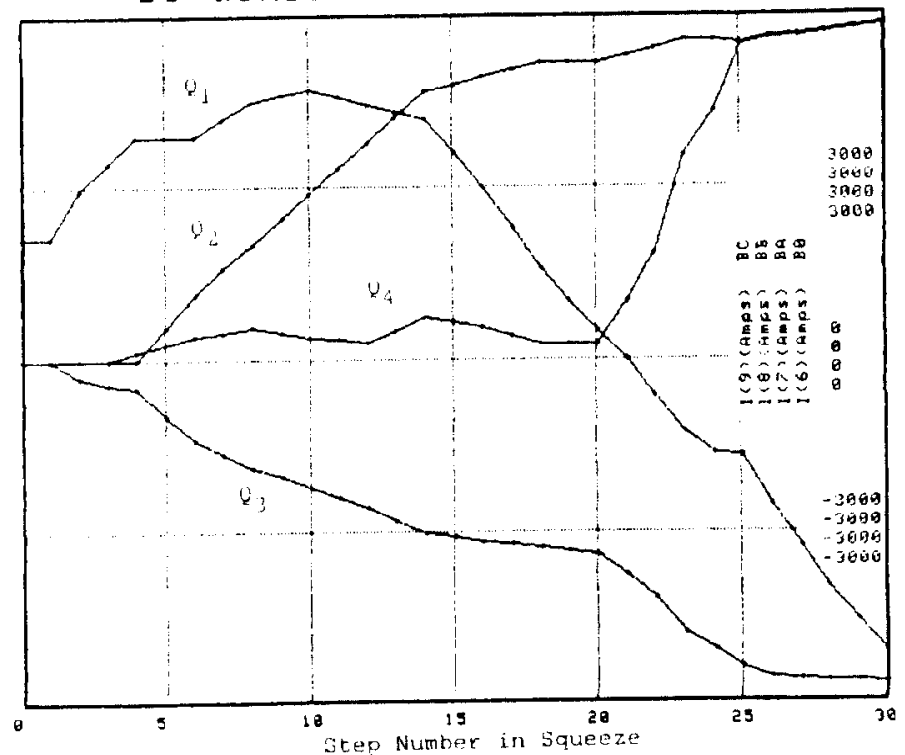

Figure 2 Current programs for the 4 new quad circuits in the adiabatic change from the fixed target lattice to the low 3 optics configuration at $1 \mathrm{TeV}$. There are 28 steps in the process. Each step takes about 2 secs and includes the simultaneous change of correction element power supplies which control the tunes, chromaticities, skew quad setting, and 20 dipole steering magnets. The total time for the "squeeze" is less than 2 minutes.

\section{Commissioning and Operation}

The computer program for the control of the function generators involved in the squeezing operation is derived from the program used to control the time and energy dependent parameters of the Tevatron. [4] In the program the low $B$ quadrupole circuits take the place of the circults which nomally are used to control the resonant extraction correction element. system for the fixed target program.

The cumbissioning procedure involves the determination of small corrections to the calculated settings for each function generator at each step. In practice, the squeeze can be stooped at any step while appropriate measurements are made and tables modified.

\section{Closed Crbit}

The Tevatron beam position monitor system [5] is used to make the necessary orbit corrections during the squeezing procedure. A special on-line computer program is used to correct the closed orbit at each of the steps in the squeeze. It uses 20 superconducting correctior dipoles in the $B O$ region to minimize the rms orbit distortion around the ring.

\section{Tunes, Coupling and Chromaticity}

A spectrum analysen is normally used to monitor the betatron tures using special resonant pickups. Smeli corrections are made to the correction element tables at each of the steps in the squeeze for tune, coupling, and chromaticity.

\section{Experience}

Transition from the injection optics has been performed during stores at constant energies of 400 $\mathrm{GeV}$ and $800 \mathrm{GeV}$. Initial problems were traced to incorrect parameters in the software. The beam turned out to be remarkably stable during the squeeze. Tune changes of $\Delta Q=0.01$ did not have a measureable impact on lifetime or beam losses. Chromaticities had to be kept positive during the squeeze to avoid collective instabilities. The closed orbit rms values were kept below $1.5 \mathrm{~mm}$. Beam losses have been observed when the rms values exceeded $3 \mathrm{~mm}$. The synchrotron frequency, which changes by about $8 \%$ due to changes in the momentum compacion factor, is not be controlled during the squeeze. After correction elements had been adjusted properiy for each of the 28 intermediate steps, a procedure which took about 2 hours, beam losses during the squeeze from $\beta=10 \mathrm{~m}$ to $0.8 \mathrm{~m}$ became negligible and the procedure could be performed routinely.

In the year that the insertion has been in place there have been about 12 study sessions where coasting beam has been studied in the low 8 configuration. The operation of the insertion has become quite routine with few incidences of beam being aborted because of equipment failure.

A remarkable feature of the insertion has been its physical stability. The original closed orbit correction tables have been sufficiently good to keep the closed orbit within $2 \mathrm{~mm}$ of the desired values for all this time. (This particular characteristic is likely to change when the heavy detector is moved into position and causes the quads to move.)

\section{Verification of Lattice Functions}

One of the first indications trat the lattice was behaying correctly was the excellent agreement between calculated and measured changes in the closed orbit when corrections were applied.

The dispersion functions around the ring were measured by comparing the closed orbits for different rf frequencies. Figure 3 shows a comparison of measured dispersion functions with a lattice calculation. Similar results have been reported for the fixed target optics.[6]

Another test to make sure that the machine behaved as predicted was the use of a flying wire scanner to measure the evolution of the beam size at each step in the squccze. The flying wire scanner is a thin $(0.05 \mathrm{~mm})$ beryllium wire which is moved quickly through the beam (from 1 to $10 \mathrm{~m} / \mathrm{s}$ depending on the desired resolution) while a downstream scintillation counter records some of the particles scattered out of the beam. Figure 4 shows the horizontal profile of the beam measured this way at the very certer of the $B O$ interaction region at successive steps in a squeeze at $400 \mathrm{GeV}$. When amplifier resolution corrections are applied, the predicted and actual changes in the beam profiles agree quite well.

\section{Conclusions}

Final conclusions on the success of the low $b$ insertion will have to wait until proton-antiproton collisions are producirg new physics results at $B O$. Nevertheless, one can say that the low $\beta$ insertion at the BO straight section is now an operational part of the Tevatron. The commissioning has evolved to the detailed verification of lattice behavior. Machine 
studies have gone on to investigations of the beam lifetime as $a$ function of hetatron tunes and chromaticties while in the low $B$ optics.

\section{Future Flans}

The first 20 steps in the squeeze are to allow the currert of the Q1 circuit to be set to 0 so that its polarity can be reversed. It has been conjectured, though not experimentally verified, that it should be possible to inject the beam at an intermediate step, say 20 , and accelerate protons and antiprotons to 800 GeV. Having stored the beam in the intermediate optics state, the final steps in the squeeze will take less time.

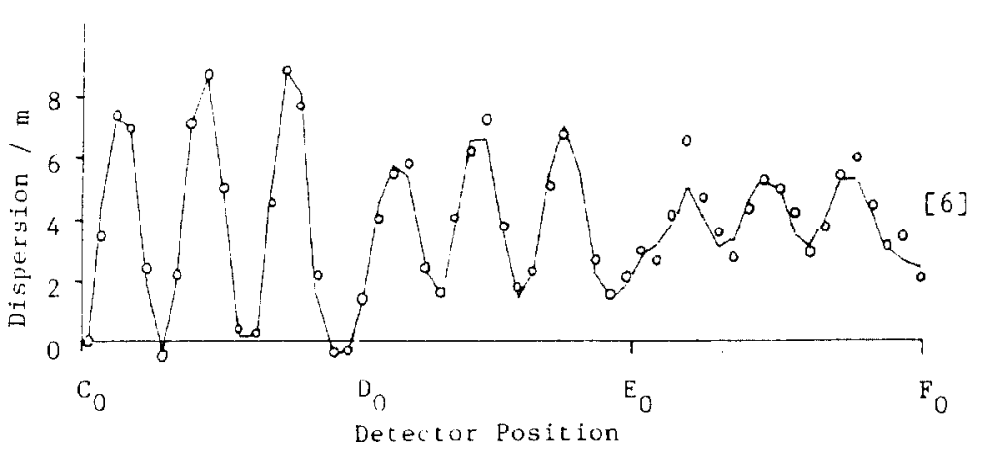

5] R. E. Shafer, R. E. Gerig,

$A$. E. Baumbaugh, and $C$. R. Wagner, "The Tevatron Beam Position And Beam Loss Monitoring Systems", in Proceedings of The 12th International Conference on High-Energy Accelerators", 1983, pp. 609-615.

6] D. A. Edwards, R. P. Johnson, and F. Willeke, "Tests of Orbital Dynamics Using The Tevatron", Fermilab-PUB-85/59 (April 1985), and submitted to Particle Accelerators.

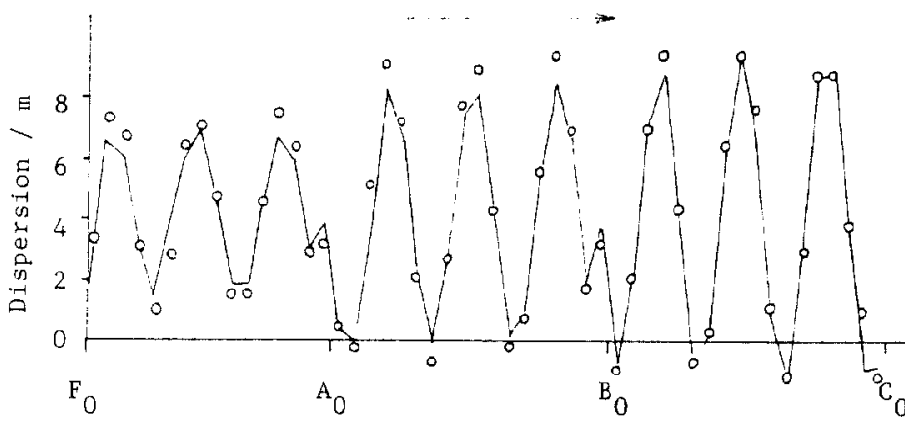

Eigure 3 Comparison of measured and predicted dispersion function for the Tevatron at $800 \mathrm{GeV}$ with the low $B$. The points are the measured values, the lines connect the predicted values at the position monitors. The abscissa is the approximate azimuthal position of the monitors.

Although the firgt tests and operation of the Tevatron with counterrotating protons and antiprotons will be with just one low $\beta$ insertion, a second one will be installed at the Do stralght section.

\section{Acknowledgements}

We thank Jim Crisp, Jeff Elseth, Lyn Evans, Jeff Gannon, and Karl Koepke for their invaluable aid in gathering the data reported above.

\section{References}

[*] Dperated by Universities Besearch Inc., under contract. with the United States Department of Energy.

[1] T. L. Collins and D. E. Johngon "A LOW-B INSERTION DESIGN FOR 1981", TM-1035, March, 1981.

D. E. Johnson, "Tevatron Bo Low $\beta$ Tuning Report, TM 1106, May 1982.

D. E. Johnson, this conference. 